\title{
XXXVIII. On the occurrence of cavitation in lubrication
}

\section{S. Skinner M.A.}

To cite this article: S. Skinner M.A. (1904) XXXVIII. On the occurrence of cavitation in lubrication, Philosophical Magazine Series 6, 7:40, 329-335, DOI: 10.1080/14786440409463123

To link to this article: http://dx.doi.org/10.1080/14786440409463123

曲 Published online: 15 Apr 2009.

Submit your article to this journal $\pi$

Џ Article views: 8

Q View related articles $\longleftarrow$

4 Citing articles: 4 View citing articles ๘ 
[Note.-These experiments were repeated during the early part of February, 1904, when the earth's orbital velocity conspires approximately with that of the solar system in space. The conditions for observation were quite as favourable as before; but no effect conld be detected. With glass, the optical system was rotated through several quadrants consecutively so as to observe any possible trace of an effect. Observations were made at noon and at 6 P.M.

Hicks*, in a more rigorous discussion than that of Lorentz, of the effect to be expected in the Michelson-Morley experiment, shows that instead of a contraction of $\frac{v^{2}}{2 V^{2}}$ in the direction of drift, there should be an elongation of $\frac{v^{2}}{2 \mathrm{~V}^{2}}$, to account for the negative results of the observations. The experiment itself should thus disprove the FitzGerald-Lorentz hypothesis. Either, on any of the suppositions possible from Lorentz's point of view, viz. contraction along the drift and zero change at right angles, no contraction but extension at right angles to the drift or elongation along and at right angles to the drift, such that the difference is $-\frac{v^{2}}{2 \mathrm{~V}^{2}}$, or, on the conclusion of Hicks, the effect to be observed by means of double refraction in the preceding experiments would be the same.-D. B. B.]

XXXVIII. On the Occurrence of Cavitation in Lubrication. By S. Skinner, M.A., University Demonstrator of Experimental Physics, Cambridge $\nmid$.

\section{[Plates XVII.-XIX.]}

$\S 1$. TTHE following experiments $\ddagger$ arose from an observation made when determining the refractive index of a liquid by means of Newton's rings. As Newton showed, the rings can be obtained when a liquid is run into the space between the lenses (Opticks, Obs. 10) ; and by comparing the radii of the rings with those obtained with the same lenses and air we have a means of measuring the refractive index between air and the liquid. The only difficulty is to arrange the illumination suitably as the rings are far fainter with the liquid than with air.

* Phil. Mag. Jan. 1902.

$\uparrow$ Communicated by the Physical Society : read November 27, 1903.

\$ These experiments formed the subject of a Demonstration before the Cambridge Philosophical Society, November 10, 1902. 
If when the liquid has been introduced the upper lens be rolled on the lower the observer sees following the central dark spot, viewed by reflected light, a crescent-shaped space, very bright provided the illumination be sufficiently oblique. This is a vacuous or vapour-filled space, for when the motion of rolling ceases the liquid flows into the space and completely fills it. Starting the rolling in the opposite direction the same appearances are seen in the reverse order. lt was the observation of this space which led me to make the following experiments, and at the time I was unaware that Newton had observed it formation, for the paragraph in which he mentions it is omitted from the quotations which have found a place in modern text-books. In Observation 11 Newton writes:- "When the Water was between the Glasses, if I pressed the upper Glass variously at its edges to make the Rings move nimbly from one place to another, a little white Spot would immediately follow the center of them, which upon creeping in of the ambient Water into that place would presently vanish. Its appearance was such as interjacent Air would have caused, and it exhibited the same Colours. But it was not Air, for where any bubbles of Air were in the Water they would not vanish. The Reflexion must have been caused by a subtiler Medium, which could recede through the Glasses at the creeping in of the Water."

$\$ 2$. The inflow of the liquid to fill the vacuous space must depend in some way on its riscosity. When a more viscous liquid is used all the effects are more pronounced, and with glycerine or lubricating oil quite large vacuous spaces, frequently broken up into a number of small bubbles, are obtained. The method of observation with oblique light takes advantage of total internal reflexion, and consequently necessitates a certain angle, depending on the refractive index of the glass, and it may be inconvenient. Another mode of observing the space is to use sodium light, when the relatively bright Newtonian rings in the space show up well in contrast with the faint rings in the liquid.

\$3. A third mode of observation, which is much the most convenient, is to use a deeply coloured liquid and to look at the space by transmitted light. I have found that a very convenient liquid is a strong solution of fuchsin in glycerine. This red solution is so deeply coloured that even up to the point where the lenses are nearest some colour shows, and that light is only brightly transmitted at the place where there is a break in the liquid. Placing this liquid between the lenses, and using daylight reflected from a sheet of white 
paper and transmitted through the system, all the appearances which are here described may be seen. It is also very convenient for photographing effects.

$\$ 4$. When the rolling ceases it has been remarked that the cavity fills up quickly with mobile, and slowly with viscons liquids, and this filling of the cavity is quite complete unless some gas or air has found its way in. The liquid is forced back to fill the vacuous space by the atmospheric pressure, and the rate of filling is decided by the viscosity of the liquid. That it is so is seen by the slowness with which oil or glycerine fills the cavity made in it, and is also evident from the relatively large size of the cavity formed in these liquids. The carvity which is formed must be produced either by splitting the liquid itself or by tearing the liquid from the glass surface. The effect mar he described as a case of "cavitation." This word has been used by the Hon. C. A. Parsons (' Nature,' May 1898) to describe the production of a cavity in water by a very rapidly rotating screw-propeller. In his experiment the atmospheric pressure was removed from the surface of the water by an airpump.

$\S 5$. Before describing the actual effects some account of the stresses in the liquid will help to make the conditions of the cavitation more clear; and in this we shall follow the graphical method used by Osborne Reynolds in hi* paper on the "Theory of Lubrication" (Phil. Trans. A. 1886). In this paper the origin of the force resisting the motion of two surfaces separated by a continuous and copious supply of lubricant is discussed, and it is shown that it is wholly accounted for by the viscosity in the lubricant.

Let $\mathrm{AB}$ and $\mathrm{CD}$ represent the sections of two parallel solid surfaces, extended infinitely at right-angles to the

Fig. 1.

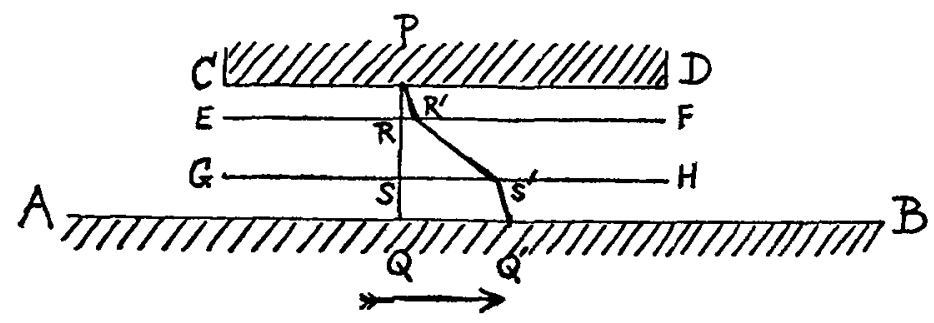

paper, and between them let us suppose three fluid layers lie, bounded by the lines $\mathrm{EF}$ and GH. Let us suppose the viscosity of the layers in contact with the solid walls is the 
same, and that the viscosity of the central layer is very much less. Let $\mathrm{PQ}$ represent $\mathrm{a}$ line in the layers when $\mathrm{AB}$ and $\mathrm{CD}$ are at rest. If now $\mathrm{AB}$ moves with a velocity $Q Q^{\prime}$, then the relative velocities of the particles in parallel layers will be represented by the displacement of PRSQ to $P R^{\prime} S^{\prime} Q^{\prime}$, $\mathrm{RR}^{\prime}$ and $\mathrm{SS}^{\prime}$ representing the velocities at the boundaries of the central layer. If, as we have supposed, the viscosity of the central laver is very small compared with that of the other layers, the inclination of $R^{\prime} S^{\prime}$ will be great, whilst $\mathrm{PR}^{\prime}$ and $\mathrm{S}^{\prime} \mathrm{Q}^{\prime}$ will be nearly parallel to $R Q$. In words, the tangential force opposing the motion of $\mathrm{AB}$ will be almost entirely due to the thin central layer of very slightly viscous fluid.

Next we consider the case where CD makes an angle with $A B$, and $E F$ and $G H$ represent as before the boundaries of the three liquid layers. It is shown by $\mathrm{O}$. Reynolds (loc. cit.)

Fig. 2.

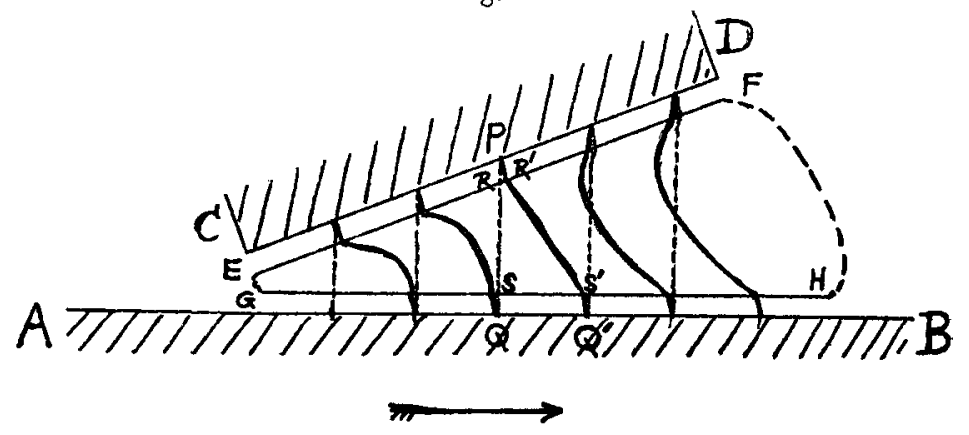

that when one of two plates, at an angle with one another, and having viscous liquid between them, $\mathrm{AB}$ is moved in the direction $\mathrm{AB}$ a tension is produced tending to draw the plates together. The line $\mathrm{PRSQ}$ becomes $\mathrm{PR}^{\prime} \mathrm{S}^{\prime} \mathrm{Q}^{\prime}$. If $\mathrm{AB}$ moves in the direction $\mathrm{BA}$ this tension becomes a pressure. Let us suppose that the ends of the lines EF and GH are joined, and then we shall have a cavity filled with less viscous fluid in a mass of viscous fluid between the two plates. Cases similar to this are treated in this paper.

$\$ 6$. In figs. 3 and 4 (Pl. XVII.) is represented a cylindrical lens lying on a plane surface with some fuchsin-glycerine solution between them. In fig. 3 everything is at rest, and the line of nearest approach of the surfaces is shown by the absence of colour, $i$. e. the bright band down the centre. In fig. 4 the cylindrical surface is being rolled by the fingers from left to right, and the transparent line of nearest approach is 
seen to have moved slightly to the right hand. Following it is seen a dark line behind which is a broader light space traversed horizontally by small streaks of dark liquid. This broader light space is filled only with the vapour of the liquid, for, as we have already stated, it is completely filled with dark liquid as soon as the motion ceases. This cavity has been formed by the splitting of the liquid, and it is of special interest to notice that it is formed not immediately at the line of nearest approach but some short distance behind this line. That the greatest tension in a lubricating liquid is not at the point of nearest approach of the surfaces was shown theoretically by Reynolds, who calculated the pressures and tensions in a layer such as this. Fig. 5 is

Fig. 5 .

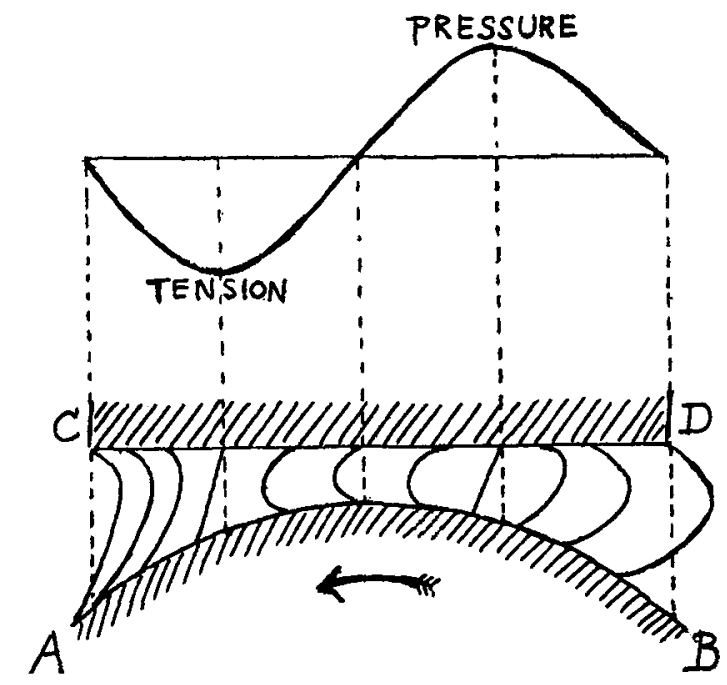

taken from Reynolds' paper, and from it we see that the point of maximum tension is behind the point of nearest approach.

$\$ 7$. In figs. 6 and 7 (Pl. XVIII.) is represented a lens lying on a plane surface. In fig. 6 everything is at rest, and the light spot at the centre shows the point of nearest approach of the lens to the plane. In fig. 7 the lens is being rolled from left to right by the fingers and a collection of cavities has been formed behind the point of nearest approach. As with the cylindrical surface, on stopping the motion the cavities fill completely.

Phil. Mag. S. 6. Vol. 7. No. 40. April 1904. 2 A 
\$ 8. In fig. 8 (Pl.XIX.) a lens is being drawn along a plane, and the cavity is visible following the motion, which is from left to right. In fig. 9 (Pl. XIX.) a biprism is being pushed from left to right, and a large cavity is visible bebind the edge of the prism which almost touches the plane. The biprism was held so that the angle between the left-hand face and the plane was greater than that between the right-hand face and the plane.

\$ 9. Some experiments were made to imitate the actual case of a fully lubricated axle rotating under a bearing. For this purpose a thick disk with its edge worked to a spherical surface of curvature equal to the radius of the disk, mounted on an axle, was arranged so that the lower part of the disk dipped into an oil-bath whilst a flat plate of glass rested on the upper edge of the disk. As the disk rotated oil was carried round so that the point of contact of the disk and plate was maintained copiously lubricated. The disk represented the axle and the plate the bearing on it. What happened on rotation could be observed by looking through the glass plate with a magnifying-glass. It was seen that during motion a cavity was formed on the side where the edge of the disk was moving away from the plate. The size of the cavity depended on the rate of rotation. When the rotation was rapid the cavity grew large and ultimately opened to the air, so that the cavity became an air-space. It was also noticed that small drops of the lubricant were carried across the cavity. These probably had their origin in the thin layer of liquid which separated the disk from the plate at their nearest approach. That there was a pressure on the one side and a tension on the other was shown by drilling a hole through the glass plate and attaching a tube to this hole. When the hole was on the approaching side of the point of nearest approach oil was forced up the tube, whilst when it was on the side where the disk's edge receded air was sucked in through the tube. In fact the latter arrangement formed a small suction-pump. The high pressure in a thoroughly well lubricated bearing was observed by Tower in his experiments on friction (Proc. Inst. Mechanical Eng. 1883-1884).

$\S 10$. It has been shown by Berthelot*, by 0 . Reynolds $\dagger$, by Worthington $\ddagger$ that a liquid can sustain a large tension or negative pressure. The ascent of sap $\$$ in tall trees has

* Ann. de Chimie et Phys. vol. xxx. p. 232 (1£50).

† Reynolds, 'Collected Papers,' vol. i.

† Phil. Trans. 1892, A. p. 355.

\$ Dixon \& Joly, Phil. Trans. 1895, B.; Askenasy, Verhandl. d. naturh. med. Vereins Heidelberg, N. F. v. (1895). 
been explained by a theory in which the liquid sap is under tension. In general the effect has been studied in liquids at rest. It may be supposed that if the movement of the cylinder on the plane in the experiment described in $\S 6$ were sufficiently slow, the negative tension would not be great enough to break the liquid but would help in drawing the liquid forward to fill the space left behind the moving surface. Experiments in which the motion is slow do not show the formation of a cavity, and may be regarded as supporting this view of the action of the tensional strength of the liquid.

\&11. The slipperiness of ice * has been attributed to the presence of a layer of lubricating water under the body pressing on the ice. The water is produced by the lowering of the freezing-point where the pressure is experienced. On this view the object glides on a liquid layer and consequently viscous friction in water takes the place of the rubbing friction between the solids. Joly shows by calcula. tion that the weight of a man concentrated on the blade of a skate is sufficient to lower the freezing-point very considerably. Reynolds, arguing from the difficulty of slipping on very cold ice, comes to a similar conclusion. I wish to point out that sliding on a liquid layer is a condition under which cavitation will occur in the liquid, and that this will aid the slipping. I find that a cavity can be seen when a convex lens is pressed strongly on ice and pushed along. A cavity of this kind may be formed behind the sliding contact of a hog-back skate, and behind that of a curlingstone.

\$12. We may now point out the influence which the formation of a cavity has on lubrication. In ball-bearings completely immersed in oil the experiments show that there must be a small cavity near the point of nearest approach of each ball to its neighbours and also to the surface on which it is running. As the friction of the bearing is the viscous friction of the oil, from the considerations in $\S 5$ it follows that it must be reduced by the formation of these cavities which are filled with relatively non-viscous vapour. In fact, if a steel ball thoroughly oiled be rolled against an oiled glass plate the cavity may be directly observed. The high lubricating property of oils owes its origin not only to their superior viscosity but also possibly to the facility with which cavities may be formed in them.

* Joly, Proe. Roy. Dublin Soc. p. 453 (1886) ; O. Reynolds, Mem. and Proc. of the Lit. and Phil. Soc. of Manchester, xliii. (1899). 


\section{Phil. Mag. Ser. 6, Vol, 7, Pl, XVII.}

Fig. 3.

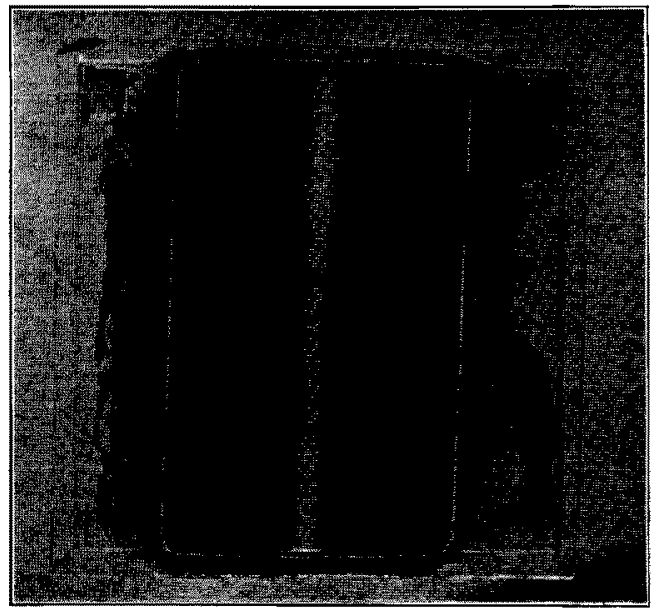

plindrical lens lying on a plane with fuchsin-glyceri solution between them. (Transmitted light.)

FIঞ. 4.

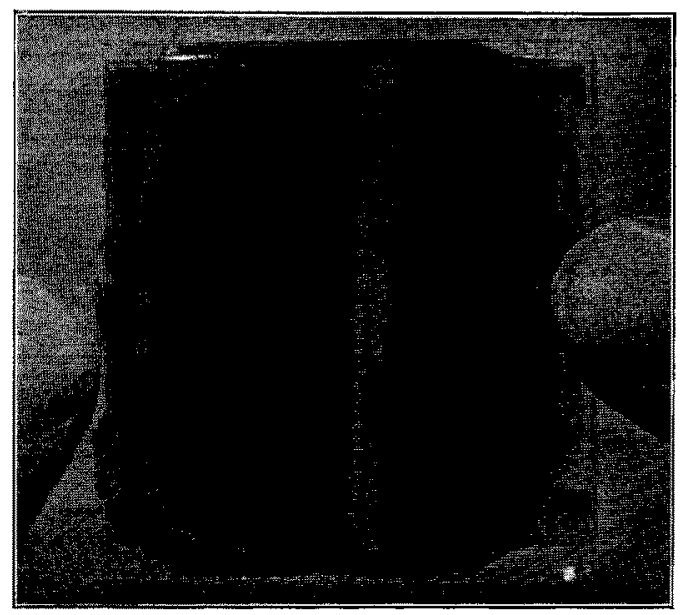

The same lens being rolled from left to right. (Transmitted and reflected light.) 


\section{Phil. Mag. Ser. 6, Vol. 7, PI. XVIII.}

FIG. 6.

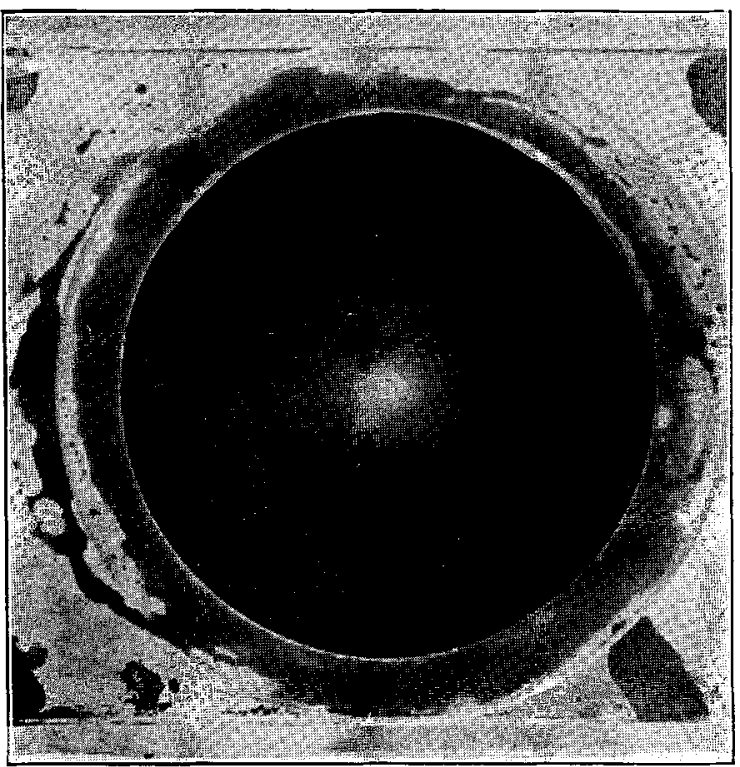

Convex lens lying on a plane with fuchsin-glycerine solution between them. (Transmitted light.)

FIG. 7.

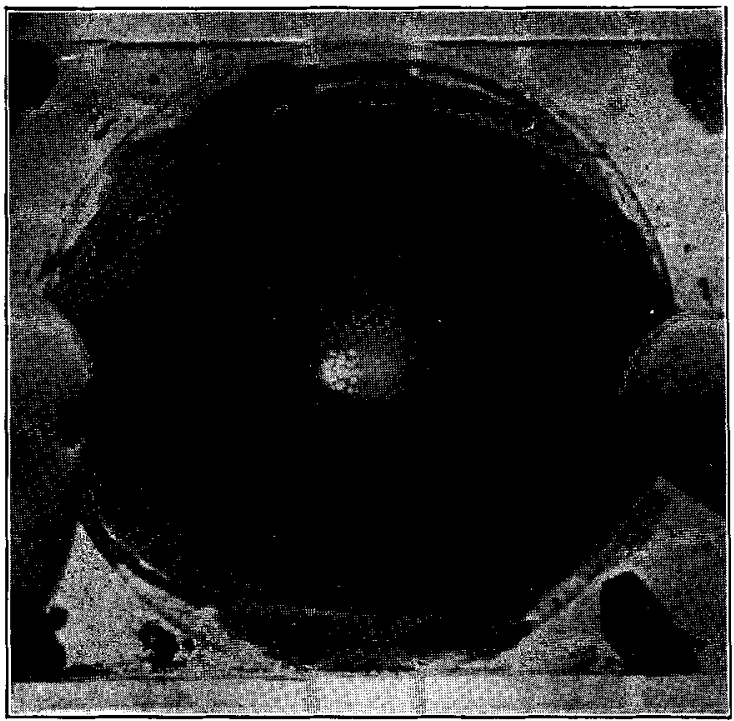

The same lens being rolled. ('Transmitted and reflected light.) 


\section{Phil, Mag. Ser. 6, Vol. 7, Pl. XIX.}

Fia. 8.

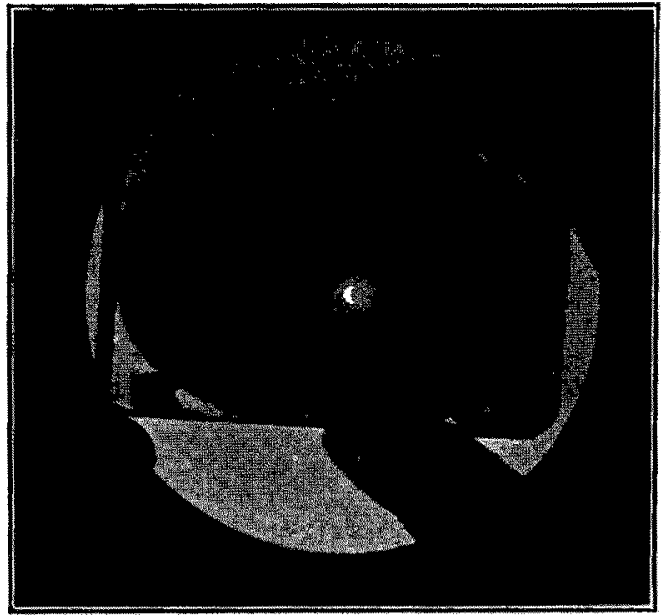

A lens being drawn along a plane from left to right. (Transmitted light.)

Fra. 9.

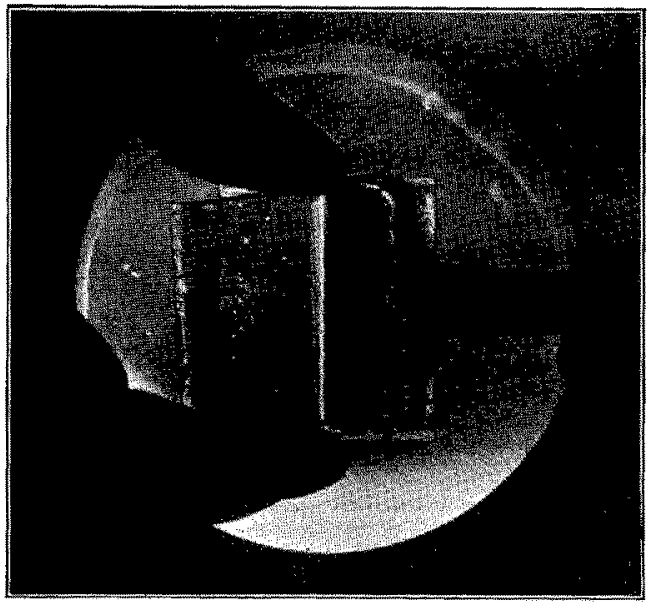

Biprism lying on a plane with fuchsin-glycerine solution between them The biprism is being pushed from left to right.

(Transmitted light.) 